2 Oudet, L. (1965). Collision and the courts. This Journal, 18, I 88.

3 Hugon, P. (1957). Les manoeuvres à faire à la mer en utilisant le radar pour eviter les abordages. Navigation (Paris) 5, 213.

\title{
A Probability Distribution for a Time-varying Quantity
}

$$
\text { D. A. Lloyd }
$$

IN many practical situations in the field of navigation, it has been noticed that the probability distribution of measured errors has a shape which has considerable departures from that of the normal distribution. These departures are particularly noticeable in the 'tails' of the distribution of practical cases, which are often higher than those of the corresponding normal distributions (see, for example, 'Is the gaussian distribution normal', W/Cdr. E. W. Anderson. This Journal, $18,65)$.

It can be shown that this phenomenon can arise from constant errors in the basic measuring device for a circuit or an apparatus containing a simple integrator. The integrator can be a real integrator or can arise from the kinematics of the situation. For example, in the case of a vehicle attempting to follow a fixed track with a heading indicator having a datum error, there is a 'kinematic integration' between heading and across-track error. In such a situation it can be shown that the error probability distribution for a large number of measurements of error is given by

$$
p(y)=\frac{1}{7 \cdot 2 \sigma_{y}}\left[-E i\left(-\frac{y^{2}}{16 \cdot 5 \sigma^{2}}\right]\right)
$$

where $p(y)$ is the probability density function for the error $y$, and $-E i(-z)$ is the exponential integral, given by

$$
-E i(-z)=\int_{z}^{\infty} \frac{e^{-q}}{q} d q
$$

If this function is plotted on linear-logarithmic graph paper, it will be seen that it can be approximated by a straight line, and this line is not very different from the straight line given by an exponential probability distribution.

The distribution is plotted on Figs. I and 2 for the case of unit variance. The curves for the normal and exponential (one sided) distributions are given for comparison, and some experimental points arising from various practical situations are plotted on the graphs to show that the curve for $p(y)$ provides a reasonable fit to a number of different practical cases. The cases plotted represent doppler drift, Loran errors, and errors from the mean of vertical speed at the entry into the flare phase of an automatic landing.

It should be noted that the 'variance' in the above formula is the time average of the variance between $o$ and $T$. The corresponding formula for $P(y)$ where $\alpha^{2} y$ is the true variance of $y$ is

$$
P(y)=\frac{1}{6 \sigma_{y}}\left[-E_{i}\left(-\frac{y^{2}}{11 \cdot 54 \sigma^{2} y}\right)\right]
$$




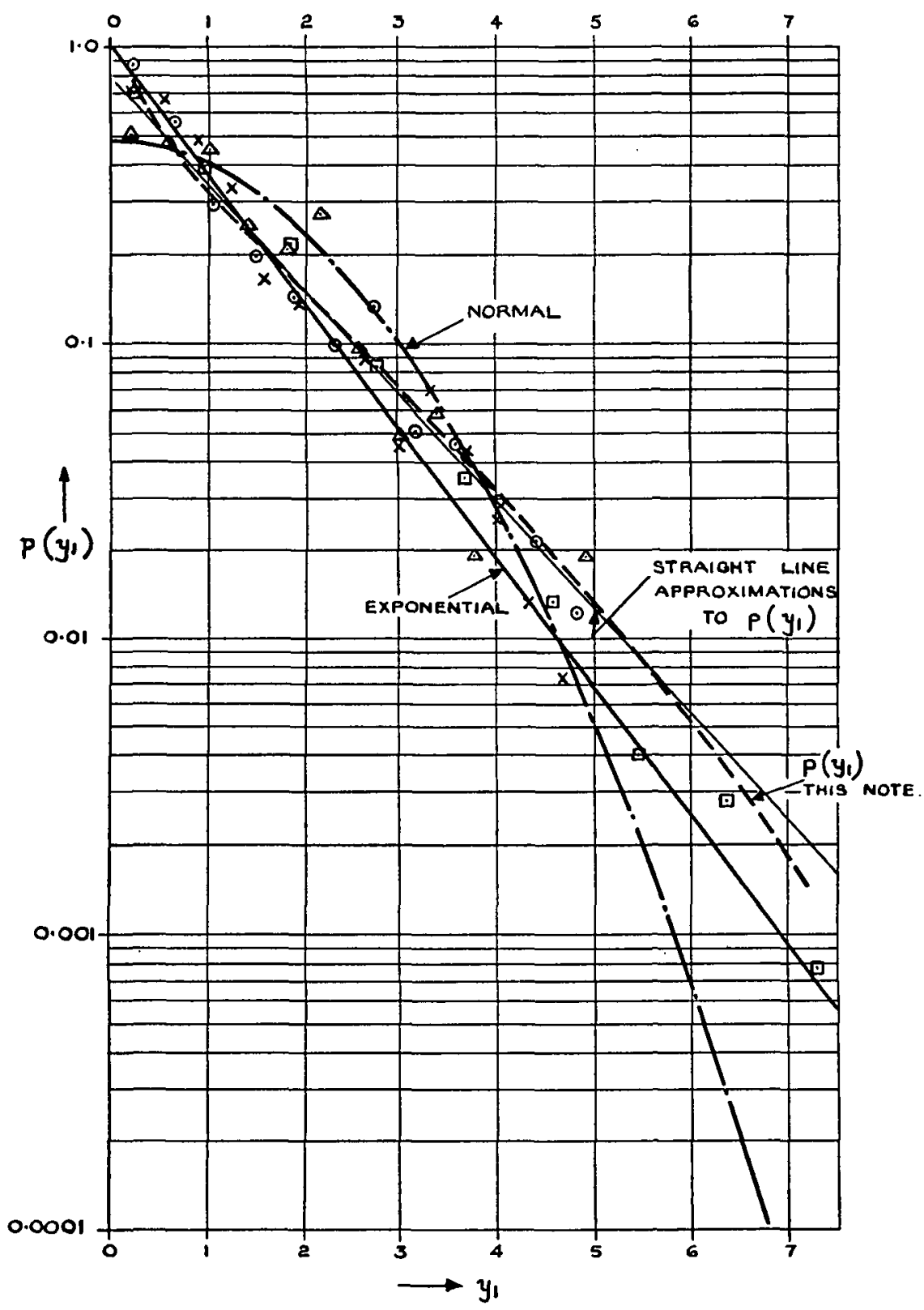

FIG. I. Probability density functions 


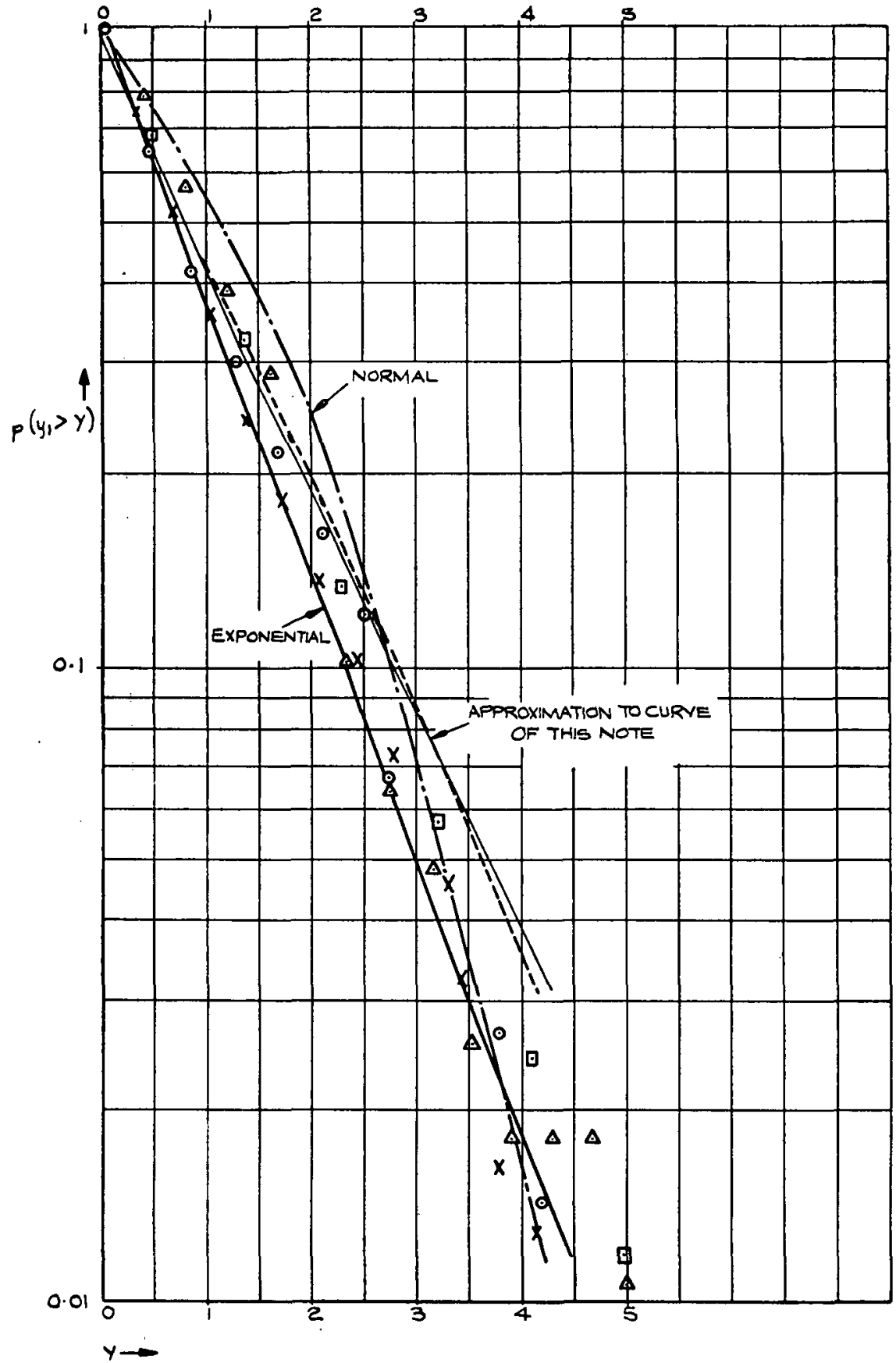

FIG. 2. Cumulative distributions 

of $y$.

This approximates closely to the exponential distribution for a wide range

The corresponding double-sided distribution is given by

$$
P(y)=\frac{1}{2 \sqrt{6 \pi \sigma_{y}}}\left[-E_{i}\left(-\frac{y^{2}}{6 \sigma^{2}}\right)\right]
$$

and in this case the difference between the time and statistical averages of the variance does not arise. This distribution also approximates closely to the exponential distribution over a range of values of $y$.

\title{
A Survey of the use of Astronomical Methods at Sea
}

\author{
D. McG. Bissett and J. J. Evans
}

THIs investigation of methods used in Astronomical Navigation has been carried out by means of questionnaires completed by senior students at a few of our Navigation Schools. The data has been classified into fairly broad categories since some of it has been rather difficult to assess, due to incomplete information on such details as trading areas, which watch was being kept, and the actual time spent at sea during the year.

The graphical analysis shows the frequency of usage for each method expressed as a percentage of the total number of observers questioned.

With regard to Solar observations the information obtained is generally as expected. The position line by Marcq. St. Hilaire is favoured a little more than Longitude by Chronometer.

(a) 42 per cent use only M. St. H. ; 27 per cent use only the longitude method; 27 per cent vary between these two methods, and only 4 per cent make use of short method tables, and

(b) of those who take ex-meridians, 9 I per cent make use of ex-meridian tables, the remainder working the calculations in full.

The Moon is noteworthy, if only for a conspicuous lack of observations, even of time azimuths. It would seem reasonable to expect that at some time of the day or night the Moon would be suitably disposed for observation, but there remains a peculiar reluctance to use it.

Considering the comparative infrequency of occasions when it is possible to observe planets, it is a little surprising to find them used so widely. Perhaps it is a matter of professional pride to the observer when he manages to locate, observe, and cross Venus with a Sun sight.

Despite a growing emphasis on the use of short method tables in the computation of zenith distance from star sights, our investigation revealed that of those regularly using stars for position fixing, 73 per cent work the calculation in full [ 5 per cent by longitude methods; $5^{8}$ per cent by $M$. St. H.] and only 27 per cent by short methods. 